MODELING, IDENTIFICATION AND CONTROL, 1998, vOL. 19, NO. 3, 141-152

do: 10.4173/mic.1998.3.2

\title{
A finite-difference method for linearization in nonlinear estimation algorithms*
}

\begin{abstract}
TOR STEINAR SCHEI $\dagger$
Keywords: Covariance matrices, estimation algorithms, extended Kalman filters, factorization methods, nonlinear filters, Jacobian matrices, linearization, parameter estimation, recursive estimation, state estimation

Linearizations of nonlinear functions that are based on Jacobian matrices often cannot be applied in practical applications of nonlinear estimation techniques. An alternative linearization method is presented in this paper. The method assumes that covariance matrices are determined on a square root factored form. A factorization of the output covariance from a nonlinear vector function is directly determined by "perturbing" the nonlinear function with the columns of the factored input covariance, without explicitly calculating the linearization and with no differentiations involved. The output covariance is more accurate than that obtained with the ordinary Jacobian linearization method. It also has an advantage that Jacobian matrices do not have to be derived symbolically.
\end{abstract}

\section{Introduction}

An extensive theory and a number of algorithms have been devcloped for nonlinear state and parameter estimation and they are based on stochastic theory. See, for example, Jazwinski (1970), Anderson and Moore (1979), and Ljung and Söderström (1983). In this theory one frequently faces the problem of approximately determining the covariance matrix for the output variables from a nonlinear vector function, based on the expected values and the covariance matrix for the input variables. The approach that is usually adopted is to determine the output covariance from the linear term (the Jacobian matrix) of a Taylor series expansion of the nonlinear function around the expected value of the input vector. Higher-order methods, which are based on higher-order terms of the Taylor series expansions and on higher-order moments of the stochastic variables, have also been proposed. However, there are important practical applications where the first- or higher-order derivatives of a nonlinear model are not well defined for certain singular values of the input variables. Methods that are based on Taylor series expansions of nonlinear functions will then fail to determine reasonable approximations for the covariance matrices.

It is well known that an alternative approach known as statistical linearization, see for example Gelb (1974), is generally more accurate than the Taylor series expansion method. However, this approach is usually computationally too demanding to be applied in practical applications.

In this paper only linear approximations to nonlinear functions will be considered for covariance calculations in nonlinear estimation problems, and higher-order methods will not be considered.

*Corresponding author: Dr Tor Steinar Schei. Tel: +47 73594375 ; Fax: + 4773594399 ; E-mail: Tor.s.schei@ecy.sintef.no.

†SINTEF Electronics and Cybernetics, 7034 Trondheim, Norway.

Received 20 August 1998.

Reprinted, with permission from Elsevier Science Ltd., from Automatica, Vol. 33, pp. 2053-2058, 1997. 
The general nonlinear vector function

$$
\mathbf{y}=\mathbf{f}(\mathbf{x})
$$

will be considered below. Equation (1) can represent a discrete state-space model, where $\mathbf{x}$ is a vector of state variables, model parameters and noise variables; and $\mathbf{y}$ is a vector of predicted states and parameters, one time-step ahead. Equation (1) can also represent an observation model, where $\mathbf{x}$ is a vector of state variables, model parameters and noise variables; and $\mathbf{y}$ is a vector of predicted measurements.

In equation (1) $\mathbf{x}$ is a vector of stochastic variables with expected value and covariance:

$$
E\{\mathbf{x}\}=\overline{\mathbf{x}}, \quad E\left\{(\mathbf{x}-\overline{\mathbf{x}})(\mathbf{x}-\overline{\mathbf{x}})^{\mathrm{T}}\right\}=X .
$$

The expected value and the covariance of $\mathbf{y}$ is determined from a linearization of $\mathbf{f}(\mathbf{x})$ around the expected value of $\mathbf{x}$ :

$$
\begin{gathered}
\overline{\mathbf{y}}=E\{\mathbf{f}(\mathbf{x})\} \approx \mathbf{f}(\overline{\mathbf{x}}), \\
Y=E\left\{(\mathbf{f}(\mathbf{x})-E\{\mathbf{f}(\mathbf{x})\})(\mathbf{f}(\mathbf{x})-E\{\mathbf{f}(\mathbf{x})\})^{\mathrm{T}}\right\} \approx F X F^{\mathrm{T}} .
\end{gathered}
$$

The matrix $F$ in equation (4) is usually determined as the Jacobian of $\mathbf{f}(\mathbf{x})$, evaluated at $\mathbf{x}=\overline{\mathbf{x}}$ :

$$
F=\left.\frac{\partial}{\partial \mathbf{x}} \mathbf{f}(\mathbf{x})\right|_{\mathbf{x}=\overline{\mathbf{x}}}
$$

There might be several reasons why Jacobian matrices are not appropriate in practical applications. First, the partial derivatives in equation (5) might not be defined for certain singular values of $\mathbf{x}$. Second, in industrial process applications $\mathbf{f}(\mathbf{x})$ might include references to a property data base, and the differentiation in equation (5) can then only be obtained numerically.

An alternative linearization method, which does not assume that the derivatives in equation (5) are defined, is proposed in the next section.

\section{New linearization method}

Initially, it is assumed that the elements $x_{j}, j=1, \ldots, n=\operatorname{dim}(\mathbf{x})$, in $\mathbf{x}$ are uncorrelated with standard deviations $\sigma_{x, j}=1 \cdot 0$. The proposed method for linearization of $\mathbf{f}(\mathbf{x})$ is then defined by:

$$
F=\{F(i, j)\}=\left\{\left(f_{i}\left(\overline{\mathbf{x}}+\mathbf{e}_{j}\right)-f_{i}\left(\overline{\mathbf{x}}-\mathbf{e}_{j}\right)\right) / 2\right\}
$$

where $F(i, j)$ is element $(i, j)$ in $F$ and $f_{i}(\cdot)$ is element $i$ in $\mathbf{f}(\cdot) . \mathbf{e}_{j}$ is the unit vector along coordinate axis $j$ in the space spanned by $\mathbf{x}$.

The difference between the linearizations in equations (5) and (6) is that element $(i, j)$ in equation (5) is the partial derivative $\partial f_{i}(\mathbf{x}) / \partial x_{j}$ at $\mathbf{x}=\overline{\mathbf{x}}$, while element $(i, j)$ in equation (6), which can be seen as a central point finite-difference approximation, is equal to the average value of $\partial f_{i}(\mathbf{x}) / \partial x_{j}$ over an interval determined by the straight line from $\overline{\mathbf{x}}-\sigma_{x, j} \mathbf{e}_{j}$ to $\overline{\mathbf{x}}+\sigma_{x, j} \mathbf{e}_{j}$. The latter explanation assumes that the partial derivatives are well defined.

The linearization equation (6) is extended to general covariance matrixes $X$ by introducing a linear transformation that statistically decouples the stochastic vector $\mathbf{x}$. Such a transformation is recognized from a square root factorization of the covariance matrix $X$ :

$$
X=S_{x} S_{x}^{\mathrm{T}}
$$


The square root matrix $S_{x}$ is not unique, but for computational reasons it is often preferred to keep it square and triangular. $S_{x}$ can be considered as a linear transformation from a stochastic vector $\mathbf{z}$ to $\mathbf{x}: \mathbf{x}=S_{x} \mathbf{z}$, where the covariance of $\mathbf{z}$ is equal to the identity matrix. Hence, by introducing the function $\tilde{\mathbf{f}}(\mathbf{z})=\mathbf{f}\left(S_{x} \mathbf{z}\right)$, equation (6) can be extended to general covariance matrices:

$$
\tilde{F}=\{\tilde{F}(i, j)\}=\left\{\left(\tilde{f}_{i}\left(\overline{\mathbf{z}}+\mathbf{e}_{j}\right)-\tilde{f}_{i}\left(\overline{\mathbf{z}}-\mathbf{e}_{j}\right)\right) / 2\right\}=F S_{x}
$$

where $\overline{\mathbf{z}}=S_{x}^{-1} \overline{\mathbf{x}}$ and $\mathbf{e}_{j}$ is the unit vector along coordinate axis $j$ in the space spanned by $z$. Hence, a square root factorization of the covariance $Y$ is determined from equations (4) and (8):

$$
\begin{aligned}
S_{y} & =\left\{S_{y}(i, j)\right\} \approx F S_{\lambda} \\
& =\left\{\left(f_{i}\left(\overline{\mathbf{x}}+\mathbf{s}_{\lambda, j}\right)-f_{i}\left(\overline{\mathbf{x}}-\mathbf{s}_{x, j}\right)\right) / 2\right\} \\
Y & =S_{y} S_{y}^{\mathrm{T}}
\end{aligned}
$$

where $\mathbf{s}_{x, j}$ is column $j$ of $S_{x}$. Equation (9) is a convenient formula for approximately determining a square root covariance $S_{y}$ without explicitly computing the linearization $F$. The accuracy of this formula is analyzed in the appendix under the assumption that $\mathbf{f}(\mathbf{x})$ is analytic. The accuracy depends on the distribution of $\mathbf{x}$, but it is always better than that obtained by using the Jacobian matrix. The improvement may be marginal. However, a main advantage with the proposed linearization method is that it can be used for linearizing models where the first derivative of $\mathbf{f}(\mathbf{x})$ is discontinuous or not defined for certain values of $\mathbf{x}$. An example of such a model is shown in Section 4.

Another advantage with the proposed method is that Jacobian matrices do not have to be derived, which may be inconvenient in connection with general estimation software which is designed to be used with arbitrary models. For example, if a model is defined by configuring various model units from a model library, it may be inconvenient to obtain the Jacobian matrix for the resulting model symbolically.

The proposed method is computationally quite efficient because it takes advantage of the sparsity of large models. The number of numerical operations necessary for evaluating $\mathbf{f}(\mathbf{x})$ is approximately proportional to $n$ rather than $n^{2}$ for large models, and the number of operations involved in equation (9) is then proportional to $n^{2}$. Another advantage with the formulation above is that the numerical properties of estimation algorithms are generally improved by using square root factorizations of covariance matrices (Carlson 1973; Bierman 1977; Thornton and Bierman 1978).

\section{Application to the extended Kalman filter}

The linearization method discussed in the previous section is applied to the extended Kalman filter.

\subsection{The extended Kalman filter $(E K F)$}

The basic Kalman filter algorithm, which is an optimal minimum variance estimator, is derived for linear systems. The theory is extended to nonlinear systems with well-defined first-order derivatives in the extended Kalman filter (EKF) algorithm, which is based on linearizations of nonlinear models around the current state estimate.

The equations for the EKF algorithm are summarized below. It is assumed that the nonlinear model is formulated in discrete time as 


$$
\begin{aligned}
\mathbf{x}(k+1) & =\mathbf{f}(\mathbf{x}(k), \mathbf{v}(k), \mathbf{u}(k)) \\
\mathbf{y}(k) & =\mathbf{g}(\mathbf{x}(k), \mathbf{w}(k))
\end{aligned}
$$

where $\mathbf{x}(k)$ is the state vector at time instant $k, \mathbf{v}(k)$ is a vector of disturbances, and $\mathbf{u}(k)$ is a vector of manipulated variables. $\mathbf{y}(k)$ is a vector of measurements and $\mathbf{w}(k)$ is a vector of measurement noisc. Both $\mathbf{f}(\cdot)$ and $\mathbf{g}(\cdot)$ can also be explicit functions of the time instant $k$.

The stochastic inputs, $\mathbf{v}(k)$ and $\mathbf{w}(k)$ are assumed to be uncorrelated white noise processes with expected values and covariances:

$$
\begin{array}{ll}
E\{\mathbf{v}(k)\}=\overline{\mathbf{v}}(k), & E\left\{(\mathbf{v}(k)-\overline{\mathbf{v}}(k))(\mathbf{v}(k)-\overline{\mathbf{v}}(k))^{\mathrm{T}}\right\}=V(k), \\
E\{\mathbf{w}(k)\}=\overline{\mathbf{w}}(k), & E\left\{(\mathbf{w}(k)-\overline{\mathbf{w}}(k))(\mathbf{w}(k)-\overline{\mathbf{w}}(k))^{\mathrm{T}}\right\}=W(k) .
\end{array}
$$

At time instant $k$, it is assumed that the a priori estimates of the state vector, $\overline{\mathbf{x}}(k)$, the measurement vector, $\overline{\mathbf{y}}(k)$, and the state covariance matrix, $\bar{X}(k)$, are determined. The equations for computation of the Kalman filter gain matrix, $K(k)$, and the a posteriori estimates of the state vector, $\hat{\mathbf{x}}(k)$, and the state covariance matrix, $\hat{X}(k)$, can be formulated as

$$
\begin{gathered}
K(k)=\bar{X}(k)\left(\frac{\partial}{\partial \mathbf{x}} \mathbf{g}\right)^{\mathrm{T}}\left[\frac{\partial}{\partial \mathbf{x}} \mathbf{g} \bar{X}(k) \frac{\partial}{\partial \mathbf{x}} \mathbf{g}^{\mathrm{T}}+\frac{\partial}{\partial \mathbf{w}} \mathbf{g} W(k) \frac{\partial}{\partial \mathbf{w}} \mathbf{g}^{\mathrm{T}}\right]^{-1}, \\
\hat{\mathbf{x}}(k)=\overline{\mathbf{x}}(k)+K(k)[\mathbf{y}(k)-\overline{\mathbf{y}}(k)], \\
\hat{X}(k)=\left[I-K(k) \frac{\partial}{\partial \mathbf{x}} \mathbf{g}\right] \bar{X}(k)\left[I-K(k) \frac{\partial}{\partial \mathbf{x}} \mathbf{g}\right]^{\mathrm{T}} \\
+\left[K(k) \frac{\partial}{\partial \mathbf{w}} \mathbf{g}\right] W(k)\left[K(k) \frac{\partial}{\partial \mathbf{w}} \mathbf{g}\right]^{\mathrm{T}} .
\end{gathered}
$$

The partial derivatives $\partial \mathbf{g} / \partial \mathbf{x}$ and $\partial \mathrm{g} / \partial \mathbf{w}$ are evaluated at the current a priori state estimate, $\mathbf{x}=\overline{\mathbf{x}}(k)$, and at $\mathbf{w}=\overline{\mathbf{w}}(k)$.

The a priori estimates are determined as

$$
\begin{gathered}
\overline{\mathbf{x}}(k+1)=\mathbf{f}(\hat{\mathbf{x}}(k), \overline{\mathbf{v}}(k), \mathbf{u}(k)), \\
\overline{\mathbf{y}}(k+1)=\mathbf{g}(\tilde{\mathbf{x}}(k+1), \overline{\mathbf{w}}(k+1)), \\
\bar{X}(k+1)=\frac{\partial}{\partial \mathbf{x}} \mathbf{f} \hat{X}(k)\left(\frac{\partial}{\partial \mathbf{x}} \mathbf{f}\right)^{\mathrm{T}}+\frac{\partial}{\partial \mathbf{v}} \mathbf{f} V(k)\left(\frac{\partial}{\partial \mathbf{v}} \mathbf{f}\right)^{\mathrm{T}} .
\end{gathered}
$$

The partial derivatives $\partial \mathbf{f} / \partial \mathbf{x}$ and $\partial \mathbf{f} / \partial \mathbf{v}$ are evaluated at the current a posteriori state estimate, $\mathbf{x}=\hat{\mathbf{x}}(k)$, and at $\mathbf{v}=\overline{\mathbf{v}}(k)$.

\subsection{Modified EKF algorithm}

The EKF algorithm is modified in accordance with equations (7) and (9). The following factorizations are introduced:

$$
\begin{array}{ll}
\bar{X}=\bar{S}_{x} \bar{S}_{x}^{\mathrm{T}}, & \hat{X}=\hat{S}_{x} \hat{S}_{x}^{\mathrm{T}}, \\
V=S_{v} S_{v}^{\mathrm{T}}, & W=S_{u} S_{u}^{\mathrm{T}} .
\end{array}
$$

$S_{v}$ and $S_{w}$ are determined from Cholesky decompositions of $V$ and $W$. 


\subsubsection{Prediction}

A square root factorization of the a priori state covariance is determined as

where

$$
\bar{S}_{x}=\left[\begin{array}{ll}
\bar{S}_{x i} & \bar{S}_{x v}
\end{array}\right]
$$

$$
\begin{aligned}
\bar{S}_{x i} & =\left\{\bar{S}_{x \hat{r}}(i, j)\right\} \\
& =\left\{\left(f_{i}\left(\hat{\mathbf{x}}+\hat{\mathbf{s}}_{x, j}, \overline{\mathbf{v}}, \mathbf{u}\right)-f_{i}\left(\hat{\mathbf{x}}-\hat{\mathbf{s}}_{x, j}, \overline{\mathbf{v}}, \mathbf{u}\right)\right) / 2\right\}, \\
\bar{S}_{x i} & =\left\{\bar{S}_{x r}(i, j)\right\} \\
& =\left\{\left(f_{i}\left(\hat{\mathbf{x}}, \overline{\mathbf{v}}+\mathbf{s}_{v, j}, \mathbf{u}\right)-f_{i}\left(\hat{\mathbf{x}}, \overline{\mathbf{v}}-\mathbf{s}_{v, j}, \mathbf{u}\right)\right) / 2\right\} .
\end{aligned}
$$

$\hat{\mathbf{s}}_{\lambda, j}$ is column $j$ of $\hat{S}_{x}$, and $\mathbf{s}_{v, j}$ is column $j$ of $S_{v}$.. Similarly, a square root factorization of the covariance

is determined as

$$
S_{y} S_{y}^{\mathrm{T}}=E\left\{(\mathbf{y}-\overline{\mathbf{y}})(\mathbf{y}-\overline{\mathbf{y}})^{\mathrm{T}}\right\}
$$

$$
S_{y}=\left[\begin{array}{ll}
S_{y \bar{x}} & S_{y w}
\end{array}\right]
$$

where $S_{y \bar{x}}$ and $S_{y w}$ are determined from equation (16), in accordance with equation (9):

$$
\begin{aligned}
S_{y \bar{x}} & =\left\{S_{y \bar{x}}(i, j)\right\} \\
& =\left\{\left(g_{i}\left(\overline{\mathbf{x}}+\overline{\mathbf{s}}_{x, j}, \overline{\mathbf{w}}\right)-g_{i}\left(\overline{\mathbf{x}}-\overline{\mathbf{s}}_{x, j}, \mathbf{w}\right)\right) / 2\right\}, \\
S_{y w} & =\left\{S_{y w}(i, j)\right\} \\
& =\left\{\left(g_{i}\left(\overline{\mathbf{x}}, \overline{\mathbf{w}}+\overline{\mathbf{s}}_{w, j}\right)-g_{i}\left(\overline{\mathbf{x}}, \overline{\mathbf{w}}+\mathbf{s}_{w, j}\right)\right) / 2\right\} .
\end{aligned}
$$

$\overline{\mathbf{s}}_{x, j}$ is column $j$ of $\bar{S}_{x}$, and $\mathbf{s}_{w, j}$ is column $j$ of $S_{\mathrm{w}}$. The rectangular matrix $\bar{S}_{x}$ in equation (19) needs to be transformed to a square matrix. This can be achieved by using the Householder transformation (see, for example, Golub and Loan 1989), which transforms $\bar{S}_{x}$ to a square and triangular matrix such that $\bar{S}_{x} \bar{S}_{x}^{T}$ is unchanged. An alternative transformation is the singular value decomposition, which also can be used to transform the rectangular matrix $\bar{S}_{x}$ to a square matrix such that $\bar{S}_{x} \bar{S}_{x}^{\mathrm{T}}$ is unchanged. The columns of $\bar{S}_{x}$ are then equal to the eigenvectors (and the singular vectors) of the covariance matrix $\bar{X}=\bar{S}_{x} \bar{S}_{x}^{\mathrm{T}}$. The singular value decomposition is, however, computationally more demanding than the Householder transformation. The Householder transformation is used in the simulation example in the next section.

\subsubsection{Filtering}

A common formulation of the equations for computation of the Kalman filter gain and the a posteriori estimates of the state vector and the state covariance matrix are shown in equations (12)-(14). The equations are reformulated below:

$$
\begin{aligned}
& K(k)=\bar{S}_{x}(k) S_{y \tilde{x}}(k)^{\mathrm{T}}\left[S_{y}(k) S_{y}(k)^{\mathrm{T}}\right]^{-1}, \\
& \hat{\mathbf{x}}(k)=\overline{\mathbf{x}}(k)+K(k)[\mathbf{y}(k)-\overline{\mathbf{y}}(k)], \\
& \hat{S}_{x}(k)=\left[\left(\bar{S}_{x}(k)-K(k) S_{y i}(k)\right) \quad K(k) S_{y w}(k)\right] .
\end{aligned}
$$

$\hat{S}_{x}(k)$ in equation (28) is rectangular, and the Householder transformation is used to transform it to a square and triangular matrix. 


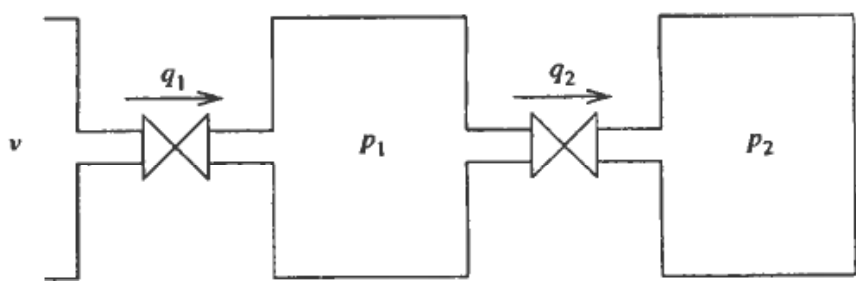

Figure 1. A system with two gas tanks (pressures $p_{1}$ and $p_{2}$ ) and two flow restrictions (flow rates $q_{1}$ and $q_{2}$ ). The pressures in the two tanks are influenced by the disturbance $v$.

\section{Simulation example}

The modified EFK algorithm is studied in a simple simulation example. A system with two gas tanks and two flow restrictions is shown in Fig. 1.

The pressures in the two tanks, $p_{1}$ and $p_{2}$, can be modelled as

$$
\begin{aligned}
& \dot{p}_{1}=p_{1}\left(q_{1}-q_{2}\right), \\
& \dot{p}_{2}=p_{2} q_{2}
\end{aligned}
$$

where $q_{1}$ and $q_{2}$ are volume flow rates as indicated in the figure. Equations (29) are in dimensionless form, and it is assumed that the differential pressures across the two flow restrictions, $v-p_{1}$ and $p_{1}-p_{2}$, are small compared to the absolute pressures $p_{1}$ and $p_{2}$. The flow rates, $q_{1}$ and $q_{2}$, are usually modelled as

$$
\begin{aligned}
& q_{1}=k_{1} \operatorname{sign}\left(v-p_{1}\right) \sqrt{\left|v-p_{1}\right|}, \\
& q_{2}=k_{2} \operatorname{sign}\left(p_{1}-p_{2}\right) \sqrt{\mid \overline{p_{1}-p_{2} \mid}}
\end{aligned}
$$

for turbulent flows across restrictions.

The pressure $v$ is a stochastic disturbance that is assumed to be uncorrelated with the states $p_{1}$ and $p_{2}$. An Euler discretization (e.g. Cheney and Kincaid, 1985) of equation (29), with time step equal one, leads to the following discrete model:

$$
\begin{aligned}
& p_{1}(k+1)=p_{1}(k)+p_{1}(k)\left(q_{1}(k)-q_{2}(k)\right), \\
& p_{2}(k+1)=p_{2}(k)+p_{2}(k) q_{2}(k) .
\end{aligned}
$$

The disturbance $v(k)$ is assumed to be white noise with expected value $\bar{v}=1.0$ and standard deviation $\sigma_{\mathrm{r}}=0.01$. The parameters in equation (30) are set by $k_{1}=k_{2}=0.01$.

The prediction part of the ordinary and the modified EKF algorithms are studied for the nonlinear model in equations (30) and (31), with no measurement update. These algorithms are compared with the results obtained from an ideal Monte Carlo analysis, where model and noise properties are identical as for the estimators above. The analysis is performed with $N=10^{4}$ run, and the stochastic disturbance, $v(k)$, has a Gaussian distribution. The expected state vector and the state covariance matrix are determined from

$$
\overline{\mathbf{p}}_{\mathrm{MC}}(k)=\frac{1}{N} \sum_{n=1}^{N} \mathbf{p}_{n}(k)
$$




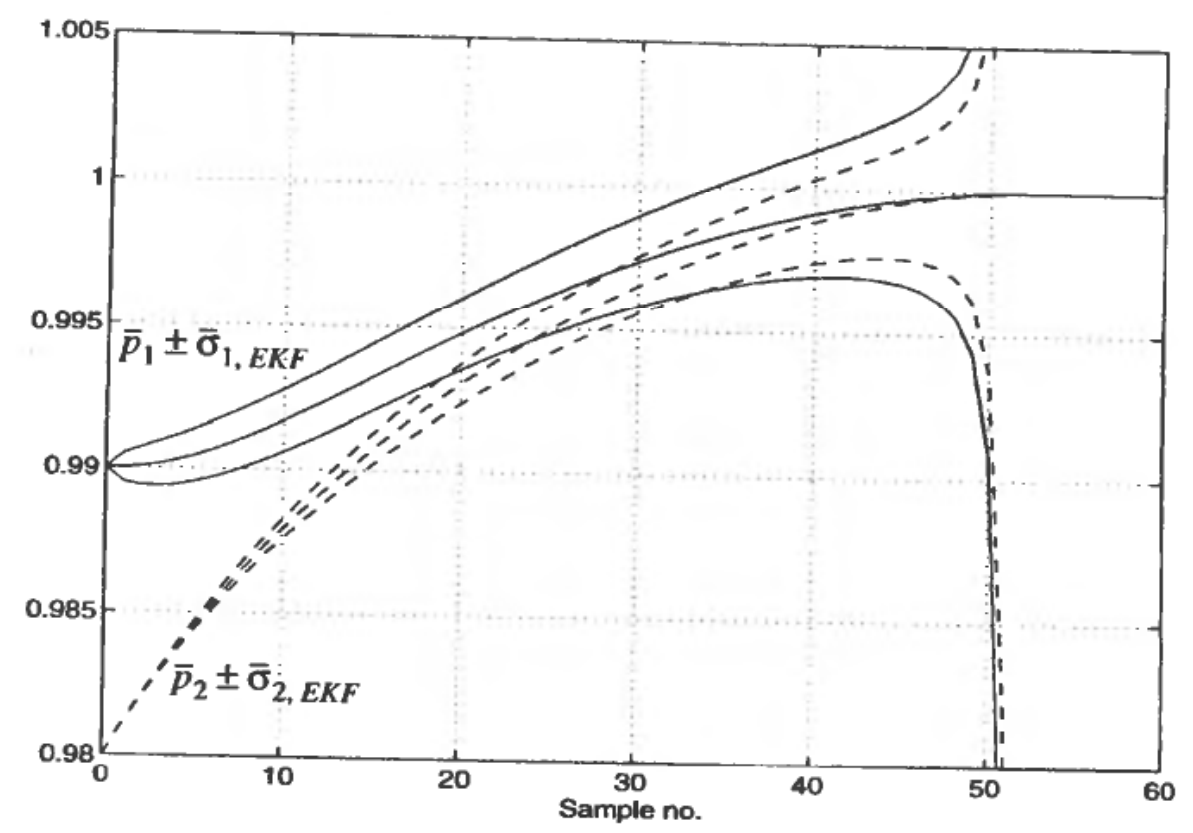

Figure 2. Jacobian linearization. The expected values $\bar{p}_{1}$ (solid line) and $\bar{p}_{2}$ (dashed line) are shown together with $\bar{p}_{1} \pm \bar{\sigma}_{1, \mathrm{EKF}}$ and $\bar{p}_{2} \pm \bar{\sigma}_{2, \mathrm{EKF}}$ where $\bar{\sigma}_{1, \mathrm{EKF}}$ and $\bar{\sigma}_{2, \mathrm{EKF}}$ are the standard devations with Jacobian linearization.

$$
\bar{P}_{\mathrm{MC}}(k)=\frac{1}{N-1} \sum_{n=1}^{N}\left(\mathbf{p}_{n}(k)-\overline{\mathbf{p}}_{\mathrm{MC}}(k)\right)^{\mathrm{T}}
$$

where $\mathbf{p}_{n}$ is the vector of pressures, $p_{1}$ and $p_{2}$, from run No. $n$. The standard deviations and correlation coefficients are then determined from

$$
\begin{gathered}
\bar{\sigma}_{1, \mathrm{MC}}=\sqrt{\bar{P}_{\mathrm{MC}}(1,1)}, \quad \bar{\sigma}_{2, \mathrm{MC}}=\sqrt{\bar{P}_{\mathrm{MC}}(2,2)}, \\
\bar{\rho}_{12, \mathrm{MC}}=\frac{\bar{P}_{\mathrm{MC}}(1,2)}{\bar{\sigma}_{1, \mathrm{MC}} \bar{\sigma}_{2, \mathrm{MC}}} .
\end{gathered}
$$

The initial states are $p_{1}(0)=0.99$ and $p_{2}(0)=0.98$, both in the estimator and for the Monte Carlo analysis, and the initial state covariance matrix is zero.

The estimators are simulated under identical conditions and compared with the Monte Carlo analysis. The result from the ordinary EKF algorithm with Jacobian linearization is shown in Fig. 2 . The standard deviations $\bar{\sigma}_{1, \mathrm{EKF}}$ and $\bar{\sigma}_{2, \mathrm{EKF}}$ approaches infinity as $\bar{p}_{2} \rightarrow \bar{p}_{1} \rightarrow \bar{v}$, which is expected since the derivatives $\partial q_{1} / \partial p_{1}, \partial q_{1} / \partial v, \partial q_{2} / \partial p_{1}$ and $\partial q_{2} / \partial p_{2}$ approach infinity. The two methods are compared in Figs. 3-5. It is clear from the figures that the ordinary EKF algorithm with Jacobian linearization cannot be applied in this situation.

\section{Conclusions}

Linearizations that are based on differentiation of nonlinear functions often cannot be applied in practical applications of nonlinear estimation techniques. An alternative linearization method, which is based on square root factorizations of covariance matrices, is proposed in this paper: A factorization of the output covariance from a 


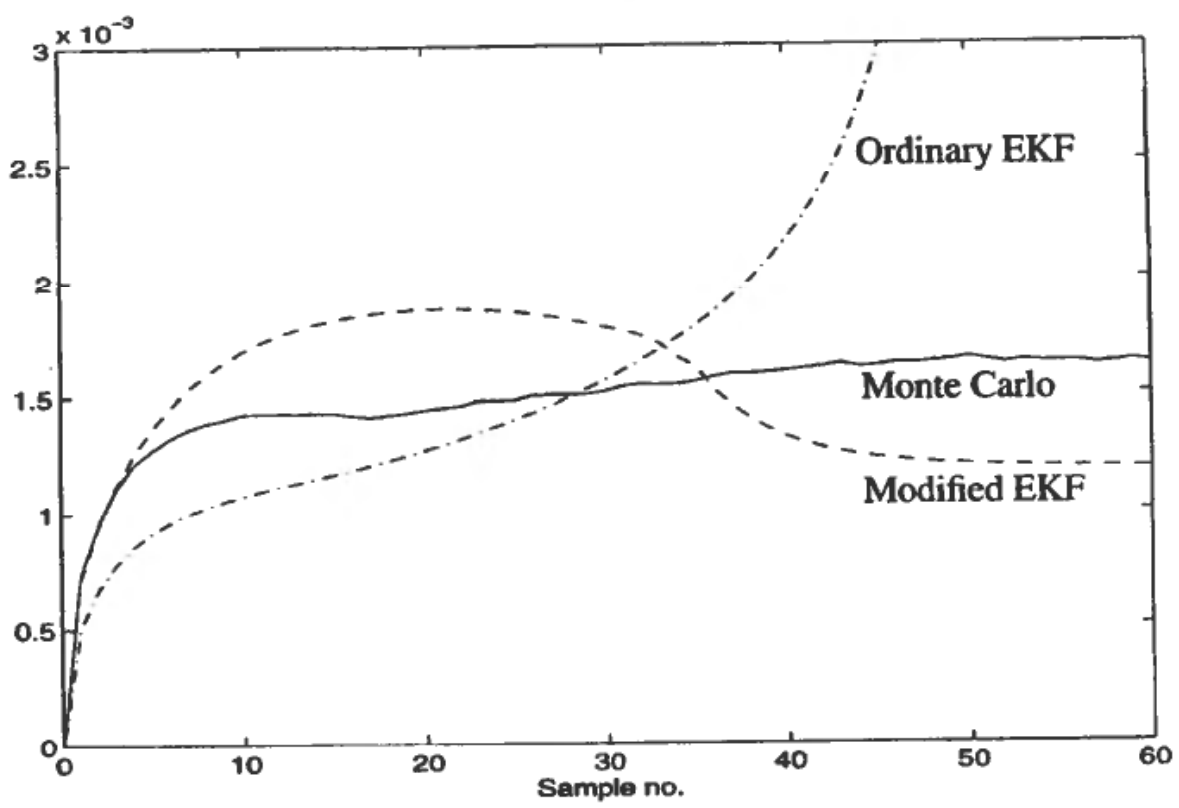

Figure 3. Standard deviation $\bar{\sigma}_{1}$.

nonlinear vector function is directly determined by "perturbing" the nonlinear function with the columns of the factored input covariance, without explicitly calculating the linearization and with no differentiations involved.

The method is more accurate than the ordinary Jacobian linearization, and the method can be applied to models where the Jacobian is not well defined. It also has the advantage that Jacobian matrices do not have to be derived symbolically. The method

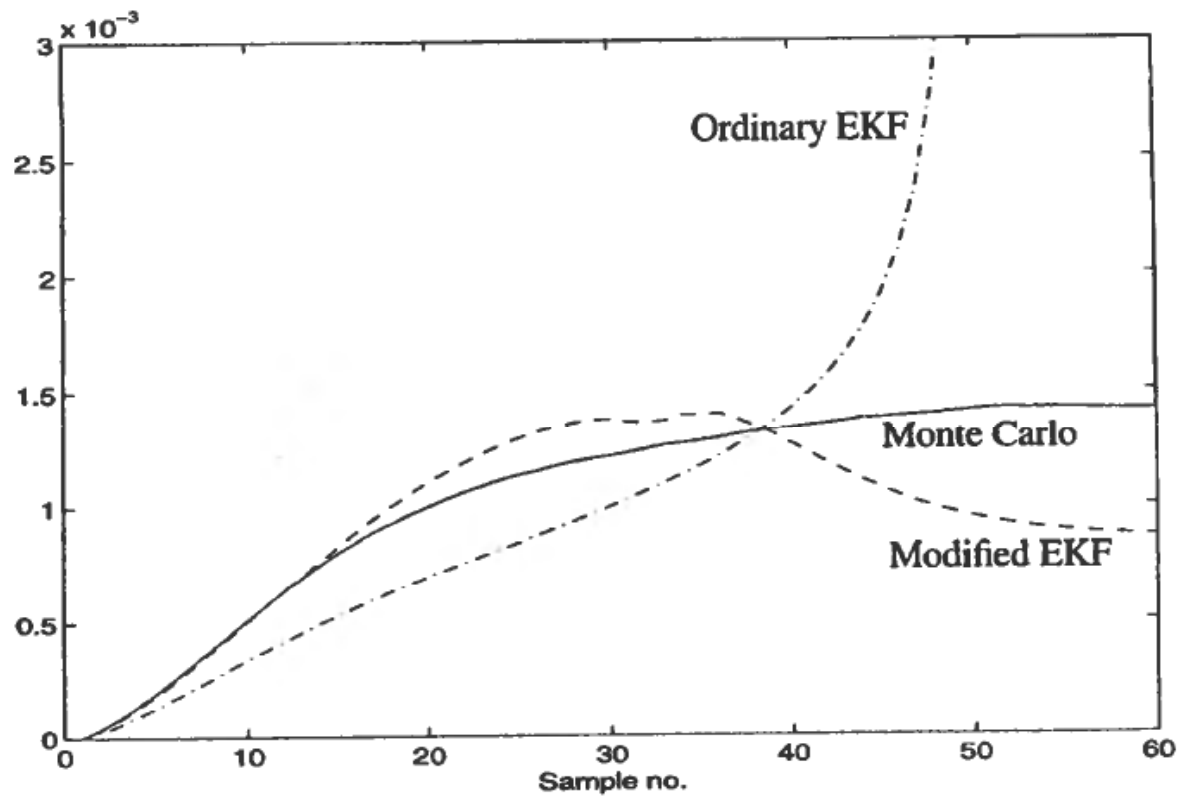

Figure 4. Standard deviation $\bar{\sigma}_{2}$. 


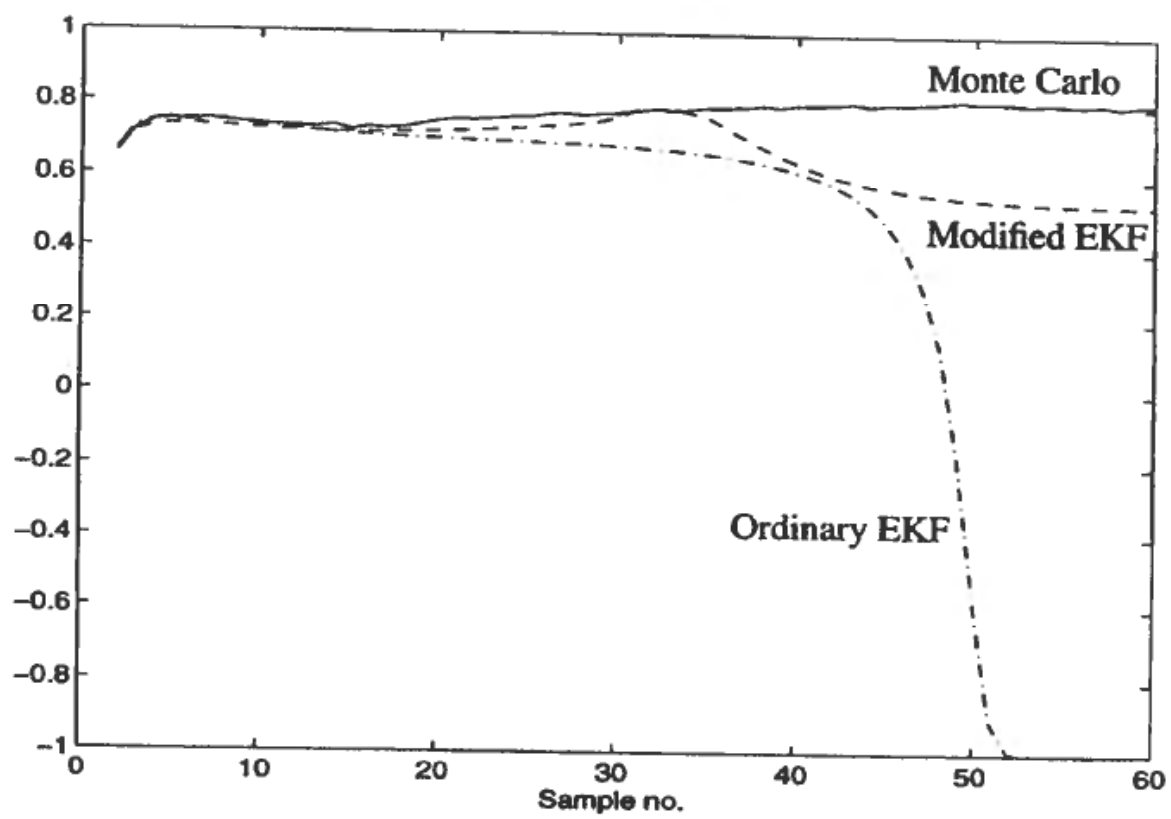

Figure 5. Correlation coefficient $\bar{\rho}_{12}$.

is particularly simple to implement, and it is numerically efficient for large models.

The proposed linearization method was studied in connection with the extended Kalman filter algorithm. The method is, however, generally applicable as an alternative to the Jacobian linearization in estimation algorithms that are based on first- and second-order moments of the stochastic variables.

\section{Appendix}

The accuracy by which the proposed linearization method in Section 2 can be used to determine approximations for covariance matrices is analyzed below. It is assumed that the expected value, $\overrightarrow{\mathbf{x}}$, and covariance, $X$, of $\mathbf{x}$ is known. For analysis purposes, it is also assumed that the distribution of $\mathbf{x}$ is Gaussian. The covariance matrix $Y$ for $\mathbf{y}=\mathbf{f}(\mathbf{x})$, determined from equation (9), is compared with the "true" covariance by expanding the nonlinear model in a multidimensional Taylor series. Hence, for the analysis it is assumed that the nonlinear function $\mathbf{f}(\mathbf{x})$ is analytic.

As in Section 2 the function $\tilde{\mathbf{f}}(\mathbf{z})=\mathbf{f}\left(S_{X} \mathbf{z}\right)$ is introduced, where $S_{x} \mathbf{z}=\mathbf{x}$ and $S_{x}$ is a quadratic square root factorization of $X, S_{x} S_{x}^{\mathrm{T}}=X$. The dimension of $\mathbf{z}$ and $\mathbf{x}$ is $n$, and the dimension of $\mathbf{y}$ is $m$. A Taylor series expansion of $\tilde{\mathbf{f}}(\mathbf{z})$ around the expected value $\overline{\mathbf{z}}=S_{x}^{-1} \overline{\mathbf{x}}$ can be expressed as

$$
\tilde{\mathbf{f}}(\mathbf{z})=\tilde{\mathbf{f}}(\overline{\mathbf{z}}+\Delta \mathbf{z})=\sum_{i=0}^{\infty} \frac{\left.D_{\Delta x}^{i}(\tilde{\mathbf{f}}(\mathbf{z}))\right|_{\mathbf{z}=\overline{\mathbf{z}}}}{i !}
$$

where the operator $D_{\Delta x}^{i}(\cdot)$, which acts on $\tilde{\mathbf{f}}(\mathbf{z})$ on a component-by-component basis, is defined as

$$
\begin{aligned}
D_{\Delta x}^{i}(\cdot) & =\left(\Delta \mathbf{z}^{\mathrm{T}} \boldsymbol{\nabla}_{\mathrm{z}}\right)^{i}(\cdot) \\
& =\left(\Delta z_{1} \frac{\partial}{\partial z_{1}}+\Delta z_{2} \frac{\partial}{\partial z_{2}}+\cdots+\Delta z_{n} \frac{\partial}{\partial z_{n}}\right)^{i}(\cdot) .
\end{aligned}
$$


The "true" expected value, $\overline{\mathbf{y}}_{T}$, is then

$$
\begin{gathered}
\overline{\mathbf{y}}_{T}=E[\tilde{\mathbf{f}}(\overline{\mathbf{z}}+\Delta \mathbf{z})]=E\left[\sum_{i=0}^{\infty} \frac{\left.D_{\Delta x}^{i}(\tilde{\mathbf{f}}(\mathbf{z}))\right|_{\mathbf{z}=\mathbf{z}}}{i !}\right] \\
=\sum_{i=0}^{\infty} \frac{\left.\left.E\left[\left(\Delta z_{1} \frac{\partial}{\partial z_{1}}+\Delta z_{2} \frac{\partial}{\partial z_{2}}+\cdots+\Delta z_{n} \frac{\partial}{\partial z_{n}}\right)^{i} \tilde{\mathbf{f}}(\mathbf{z})\right)\right|_{\mathbf{z}=\overline{\mathbf{z}}}\right]}{i !} .
\end{gathered}
$$

The elements of $\Delta \mathbf{z}$ are uncorrelated with zero expectations and equal, symmetric distribution. Equation (A.3) can then be simplified

$$
\overline{\mathbf{y}}_{T}=\tilde{\mathbf{f}}(\overline{\mathbf{z}})+\sum_{i=1}^{\infty} \frac{\left.\left(\frac{\partial^{2 i}}{\partial z_{1}^{2 i}}+\frac{\partial^{2 i}}{\partial z_{2}^{2 i}}+\cdots+\frac{\partial^{2 i}}{\partial z_{n}^{2 i}}\right) \tilde{\mathbf{f}}(\mathbf{z})\right)\left.\right|_{\mathbf{z}=\overline{\mathbf{z}}} E\left[\Delta z^{2 i}\right]}{(2 i) !}
$$

where $\Delta z$ is an arbitrary element in $\Delta \mathbf{z}$. All odd moments of $\Delta z$ are zero because the distribution is symmetric.

Element number $k$ in $\mathbf{y}-\overline{\mathbf{y}}_{T}$ can now be expressed as

$$
\begin{aligned}
y_{k}-\tilde{y}_{k, T} & =\tilde{f}_{k}(\overline{\mathbf{z}}+\Delta \mathbf{z})-E\left[\tilde{f}_{k}(\overline{\mathbf{z}}+\Delta \mathbf{z})\right]=\sum_{i=1}^{\infty} \frac{\left.\left(\Delta \mathbf{z}^{\mathrm{T}} \boldsymbol{\nabla}_{\mathbf{z}}\right)^{i}\left(\tilde{f}_{k}(\mathbf{z})\right)\right|_{\mathbf{z}=\overline{\mathbf{z}}}}{i !} \\
& -\sum_{i=1}^{\infty} \frac{\left.\left(\frac{\partial^{2 i}}{\partial z_{1}^{2 i}}+\frac{\partial^{2 i}}{\partial z_{2}^{2 i}}+\cdots+\frac{\partial^{2 i}}{\partial z_{n}^{2 i}}\right)\left(\tilde{f}_{k}(\mathbf{z})\right)\right|_{\mathbf{z}=\overline{\mathbf{z}}} E\left[\Delta z^{2 i}\right]}{(2 i) !}
\end{aligned}
$$

and clement $(k, l)$ of the true covariance matrix $Y_{T}$ is

$$
\begin{aligned}
Y_{T}(k, l)= & E\left[\left(y_{k}-\bar{y}_{k . T}\right)\left(y_{l}-\bar{y}_{l, T}\right)\right] \\
= & E\left[\left(\sum_{i=1}^{\infty} \frac{\left.\left.\left(\Delta \mathbf{z}^{\mathrm{T}} \nabla_{\mathbf{z}}\right)^{i}\left(\tilde{f}_{k}(\mathbf{z})\right)\right|_{\mathbf{z}=\overline{\mathbf{z}}}\right)}{i !}\right) \sum_{j=1}^{\infty} \frac{\left.\left.\left.\left(\Delta \mathbf{z}^{\mathrm{T}} \nabla_{\mathbf{z}}\right)^{j}\left(\tilde{f}_{l}(\mathbf{z})\right)\right|_{\mathbf{z}=\overline{\mathbf{z}}}\right)\right]}{j !}\right] \\
& -E\left[\sum_{i=1}^{\infty} \frac{\left.\left(\Delta \mathbf{z}^{\mathrm{T}} \boldsymbol{\nabla}_{\mathbf{z}}\right)^{i}\left(\tilde{f}_{k}(\mathbf{z})\right)\right|_{\mathbf{z}=\overline{\mathbf{z}}}}{i !}\right] \\
& \times\left(\sum_{j=1}^{\infty} \frac{\left.\left(\sum_{p=1}^{n} \frac{\partial^{2 j}}{\partial z_{p}^{2 j}}\right)\left(\tilde{f}_{l}(\mathbf{z})\right)\right|_{\mathbf{z}=\overline{\mathbf{z}}} E\left[\Delta z^{2 j}\right]}{(2 j) !}\right) \\
& -\left(\sum_{i=1}^{\infty} \frac{\left.\left(\sum_{p=1}^{n} \frac{\partial^{2 i}}{\partial z_{p}^{2 i}}\right)\left(\tilde{f}_{k}(\mathbf{z})\right)\right|_{\mathbf{z}=\overline{\mathbf{z}}} E\left[\Delta z^{2 i}\right]}{(2 i) !}\right) \\
& \times E\left[\sum_{j=1}^{\infty} \frac{\left.\left.\left(\Delta \mathbf{z}^{\mathrm{T}} \nabla_{\mathbf{z}}\right)^{j}\left(\tilde{f}_{l}(\mathbf{z})\right)\right|_{\mathbf{z}=\overline{\mathbf{z}}}\right]}{j !}\right) \\
& +\left(\sum_{i=1}^{\infty} \frac{\left.\left(\sum_{p=1}^{n} \frac{\partial^{2 i}}{\partial z_{p}^{2 i}}\right)\left(\tilde{f}_{k}(\mathbf{z})\right)\right|_{\mathbf{z}=\overline{\mathbf{z}}} E\left[\Delta z^{2 i}\right]}{(2 i) !}\right) \\
& \times\left(\sum_{j=1}^{\infty} \frac{\left.\left(\sum_{p=1}^{n} \frac{\partial^{2 j}}{\partial z_{p}^{2 j}}\right)\left(\tilde{f}_{i}(\mathbf{z})\right)\right|_{\mathbf{z}=\overline{\mathbf{z}}} E\left[\Delta z^{2 j}\right]}{(2 j) !}\right) .
\end{aligned}
$$


The last two terms in equation (A.6) cancel, and $Y_{T}(k, l)$ is further simplified:

$$
\begin{aligned}
Y_{T}(k, l)= & \sum_{p=1}^{n}\left(\sum_{i=1}^{\infty} \sum_{j=1}^{\infty} \frac{\left(\left.\frac{\partial^{i}}{\partial z_{p}^{i}} \tilde{f}_{k}(\mathbf{z})\right|_{\mathbf{z}=\overline{\mathbf{z}}}\right)}{i !} \frac{\left(\left.\frac{\partial^{j}}{\partial z_{p}^{j}} \tilde{f}_{l}(\mathbf{z})\right|_{\mathbf{z}=\overline{\mathbf{z}}}\right)}{j !} E\left[\Delta z^{i+j}\right]\right) \\
& -\sum_{p=1}^{n}\left(\left(\sum_{i=1}^{\infty} \frac{\left(\left.\frac{\partial^{2 i}}{\partial z_{p}^{2 i}} \tilde{f}_{k}(\mathbf{z})\right|_{\mathbf{z}=\overline{\mathbf{z}}}\right) E\left[\Delta z^{2 i}\right]}{(2 i) !}\right)\right. \\
& \left.\times\left(\sum_{j=1}^{\infty} \frac{\left(\left.\frac{\partial^{2 i}}{\partial z_{p}^{2 i}} \tilde{f}_{i}(\mathbf{z})\right|_{\mathbf{z}=\overline{\mathbf{z}}}\right) E\left[\Delta z^{2 j}\right]}{(2 j) !}\right)\right)
\end{aligned}
$$

The terms containing odd derivatives are separated from the terms with even derivatives:

$$
\begin{aligned}
Y_{T}(k, l)= & \sum_{p=1}^{n}\left(\sum_{i=0}^{\infty} \sum_{j=0}^{\infty} \frac{\left(\left.\frac{\partial^{(2 i+1)}}{\partial z_{p}^{2 i+1}} \tilde{f}_{k}(\mathbf{z})\right|_{\mathbf{z}=\bar{z}}\right)}{(2 i+1) !} \frac{\left(\left.\frac{\partial^{(2 j+1)}}{\partial z_{p}^{2 j+1}} \tilde{f}_{i}(\mathbf{z})\right|_{\mathbf{z}=\overline{\mathbf{z}}}\right)}{(2 j+1) !} E\left[\Delta z^{2 i+2 j+2}\right]\right) \\
& +\sum_{p=1}^{n}\left(\sum_{i=1}^{\infty} \sum_{j=1}^{\infty} \frac{\left(\left.\frac{\partial^{2 i}}{\partial z_{p}^{2 i}} \tilde{f}_{k}(\mathbf{z})\right|_{\mathbf{z}=\overline{\mathbf{z}}}\right)}{(2 i) !} \frac{\left(\left.\frac{\partial^{2 j}}{\partial z_{p}^{2 j}} \tilde{f}_{l}(\mathbf{z})\right|_{z=\bar{z}}\right)}{(2 j) !}\right. \\
& \left.\times\left(E\left[\Delta z^{2 i+2 j}\right]-E\left[\Delta z^{2 i}\right] E\left[\Delta z^{2 j}\right]\right)\right) .
\end{aligned}
$$

For a Gaussian distribution the even moments of $\Delta \mathrm{z}$ are (Papoulis 1984):

$$
E\left[\Delta z^{2 q}\right]=1 \cdot 3 \cdot \ldots \cdot(2 q-1) \sigma_{\Delta z}^{2 u}, \quad \sigma_{\Delta z}=1 \cdot 0 .
$$

The covariance detcrmined by using the proposed linearization method is derived from equations (8) and (9). Element $(k, l)$ of the covariance matrix is determed as

where

$$
Y(k, l)=\sum_{p=1}^{n} S_{y}(k, p) S_{y}(l, p)
$$

$$
\begin{aligned}
S_{y}(k, p) & =\frac{1}{2}\left(\tilde{f}_{k}\left(\mathbf{z}+\mathbf{e}_{p}\right)-\tilde{f}_{k}\left(\overline{\mathbf{z}}-\mathbf{e}_{p}\right)\right) \\
& =\frac{1}{2}\left(\left.\sum_{i=0}^{\infty}\left(\frac{\left(\mathbf{e}_{p}{ }^{\mathrm{T}} \nabla_{z}\right)^{i}}{i !}-\frac{\left(-\mathbf{e}_{p}{ }^{\mathrm{T}} \nabla_{z}\right)^{i}}{i !}\right)\left(\tilde{f}_{k}(\mathbf{z})\right)\right|_{\mathbf{z}=\overline{\mathbf{z}}}\right) \\
& =\sum_{i=0}^{\infty}\left(\frac{\left.\left(\mathbf{e}_{p}{ }^{\mathrm{T}} \nabla_{z}\right)^{(2 i+1)}\left(\tilde{f}_{k}(\mathbf{z})\right)\right|_{\mathbf{z}=\overline{\mathbf{z}}}}{(2 i+1) !}\right)=\sum_{i=0}^{\infty}\left(\frac{\left.\frac{\partial^{(2 i+1)}}{\partial z_{p}^{2 i+1}} \tilde{f}_{k}(\mathbf{z})\right|_{\mathbf{z}=\overline{\mathbf{z}}}}{(2 i+1) !}\right)
\end{aligned}
$$

Using equations (A.10) and (A.11) an expression for clement $(k, l)$ of the covariance matrix is obtained:

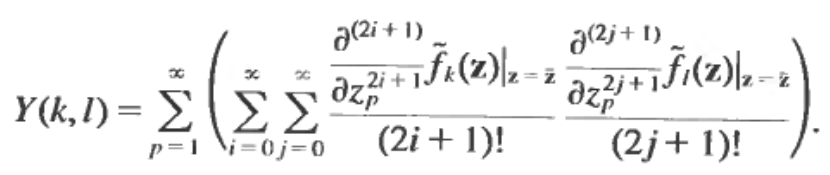


By comparing the true covariance in equations (A.8) and (A.9) with the covariance determined in equation (A.12) and the covariance determined by the ordinary Jacobian linearization method, which corresponds to using only the first derivatives in equation (A.12) $(i=0, j=0)$, it is clear that the proposed method is slightly more accurate than the Jacobian linearization method. The two methods coincide if all odd derivatives, except the first derivatives, of $\tilde{\mathbf{f}}(\mathbf{z})$ are zero, otherwise the new method is more accurate. It is also worth noting that equation (A.12) would determine the true covariance for a symmetric distribution where the even moments of $\Delta z$ are:

$$
E\left[\Delta z^{2 q}\right]=\sigma_{\Delta z}^{2 q}=1.0 .
$$

\section{REFERENCES}

ANderson, B. D. O. and Moore, J. B. (1979). Optimal Filtering. Prentice-Hall, Englewood Cliffs, NJ.

BIERMAN, G. J. (1977). Factorization Methods for Discrete Sequential Estimation. Academic Press, New York.

CARLSON, N. A. (1973). Fast triangular formulation of the square root filter. A.I.A.A.J., 11, $1259-1265$.

CHENEY, W. and KINCAID, D. (1985). Numerical Mathematics and Computing. Brooks/Cole, Monterey, CA.

GELB, A. (1974). Applied Optimal Estimation. The MIT Press, Cambridge, MA.

GoluB, G. H. and VAN LOAN, C. F. (1989). Matrix Computations. North Oxford Academic, London.

JAZWINSKI, A. H. (1970). Stochastic Processes and Filtering Theory. Academic Press, New York. LJUNG, L. and SOODERSTRÖM, T. (1983). Theory and Practice of Recursive Identification. MIT Press, Cambridge, MA.

PAPOULIS, A. (1984). Probability, Random Variables, and Stochastic Processes. McGraw-Hill, Singapore.

THORNTON, C.L. and BIERMAN, G. J. (1978). Filtering and error analysis via the UDU ${ }^{\mathrm{T}}$ covariance factorization. IEEE Trans. Automat. Control, AC-23, 901-907. 